\title{
Profil spermiologique des hommes infertiles au Mali
}

\author{
Mahamadou TRAORE, Amadou TOURE, Sidi SISSOKO, N'Dia Fatoumata SAMAKE
}

Service de Cytogénétique et de Biologie de la Reproduction

Institut National de Recherche en Santé Publique, Bamako, Mali

\section{RESUME}

L'infertilité reste un problème socioculturel très important quand on sait qu'en Afrique en général, et particulièrement au Mali, le but principal du mariage reste la procréation. La naissance d'un enfant dans un foyer est une source de joie et contribue au maintien d'une certaine harmonie dans le ménage.

En Afrique, l'infertilité constitue un drame social et une épreuve difficile à surmonter pour le couple. Elle est la première cause de mésentente conjugale ou de divorce, elle est considérée comme une fatalité, un sort ou une malédiction infligée au couple. Pour la femme, elle représenterait une punition divine car la plupart du temps, elle est la première à être mise en cause. C'est au cours d'un bilan d'infertilité du couple que la responsabilité masculine sera découverte.

Le spermogramme et le spermocytogramme sont les examens de base de la spermiologie. Ces examens constituent des éléments clés permettant d'évaluer la qualité du sperme. Au Mali, les examens spermiologiques sont effectués dans le laboratoire de Cytogénétique et de Biologie de la Reproduction, le laboratoire qui effectue le plus grand nombre d'examens de sperme dans le pays.

L'objet de ce travail est de présenter le profil spermiologique des hommes infertiles au Mali à travers une étude rétrospective sur cinq ans. L'infertilité masculine est un problème de santé publique au Mali. La tranche d'âge la plus touchée se situe entre 30 et 39 ans. La stérilité primaire est fortement représentée par $70 \%$ des sujets étudiés. La bilharziose occupe une place prédominante dans les antécédents infectieux. Les résultats du spermogramme montrent que les cas d'oligo-asthéno-zoospermie et d'azoospermie représentent respectivement $49,5 \%$ et $23,7 \%$ de la population étudiée.

Mots-clés : spermogramme, infertilité masculine, antécédents infectieux, azoospermie

\section{INTRODUCTION}

L'infertilité reste un problème socioculturel très important quand on sait qu'en Afrique en général et particulièrement au Mali, le but principal du mariage reste la procréation. La naissance d'un enfant dans un foyer est une source de joie et contribue au maintien d'une certaine harmonie dans le ménage. Les hommes s'unissent avec les femmes pour la génération d'un nouvel être humain. Cette faculté de se reproduire est assurée par leur système génital dont le fonctionnement normal implique le bon déroulement de la reproduction humaine. Malheureusement, des anomalies ou des pathologies peuvent l'affecter entraînant ainsi des troubles de son fonctionnement et rendant le couple infertile ou stérile $[4,6]$.

En Afrique, l'infertilité constitue un drame social et une épreuve difficile à surmonter pour le couple. Première cause de mésentente conjugale ou de divorce, elle est considérée comme une fatalité, un sort ou une malédiction infligée au couple. Pour la femme, elle représenterait une punition divine car la plupart du temps, elle est la première à être mise en cause $[4,5]$. Le fardeau reste lourd pour les hommes d'être mis en cause dans un problème d'infertilité du couple d'où leur faible implication dans la recherche d'une solution à ce problème. On associe encore volontiers les infertilités à la féminité, alors que les connaissances actuelles permettent de mettre en cause également homme et femme.

C'est au cours d'un bilan d'infertilité du couple que la responsabilité masculine sera découverte. Si l'examen clinique et l'interrogatoire peuvent préciser les défauts anatomiques, les troubles de l'érection ou ceux de l'éjaculation, ils sont quelques fois insuffisants et doivent

Correspondance :

Dr Mahamadou TRAORE - Service de Cytogénétique et de Biologie de la Reproduction, INRSP, BP 1771, Bamako, Mali - Tel (223) 6723208 - Email seybatraore@yahoo.fr 
être obligatoirement être complétés par l'analyse du sperme. Les techniques de biologie de la reproduction ont montré qu'il pouvait exister des spermes avec des paramètres anormaux qui étaient fécondants et inversement des spermes avec des paramètres normaux qui ne l'étaient pas [9].

Au Mali, les examens cytospermiologiques sont effectués dans le laboratoire de Cytogénétique et de Biologie de la Reproduction à I'Institut National de Recherche en Santé Publique (INRSP) depuis bientôt deux décennies $[7,8,16]$. C'est le laboratoire qui effectue le plus grand nombre d'examens de sperme dans le pays. Ces examens sont demandés par des gynécologues, des urologues et des médecins généralistes. II y a vingt ans, nous effectuions seulement une dizaine d'examens, actuellement nous en faisons une centaine par mois. Cette augmentation est due aux efforts de sensibilisation au niveau des médecins prescripteurs mais surtout au niveau des malades.

L'objectif de ce travail est de présenter le profil spermiologique des hommes infertiles au Mali à travers une étude rétrospective sur cinq ans.

\section{MATERIEL ET METHODES}

Nous présentons les résultats d'une étude rétrospective concernant 2250 dossiers de patients chez qui l'examen de sperme a été réalisé sur une période de cinq ans (20032007). Ces patients nous ont été adressés par des gynécologues, urologues, andrologues et médecins généralistes des différentes structures sanitaires publiques et privées du pays. Pour chaque dossier, nous avons tenu compte des variables suivantes : âge, statut matrimonial, type de stérilité, antécédents infectieux et médicaux, analyse du sperme (volume de l'éjaculat, numération et mobilité des spermatozoïdes), spermoculture, dosages hormonaux (FSH, $\mathrm{LH}$, Prolactine, Testostérone). Les taux d'hormones ont été déterminés par la méthode immunoenzymatique (ELFA) [21].

Le recueil de sperme a lieu par masturbation après 3 à 5 jours d'abstinence sexuelle, sur place au laboratoire ou au domicile, et dans ce dernier cas l'échantillon nous a été apporté dans l'heure qui suivait le recueil. II faut noter que pour des raisons socioculturelles, la masturbation est très mal admise dans notre société. Ceci représente une des raisons de la réticence des hommes à faire cet examen.

Les analyses spermiologiques ont été réalisées sur la base des critères de l'Organisation Mondiale de la Santé [22]. L'étude du sperme n'a été entreprise qu'avec toutes les garanties que le prélèvement a été fait dans les conditions requises à cet effet. Nous avons définie l'oligozoospermie comme étant une numération de spermatozoïdes inférieure à $20 \times 106 / \mathrm{ml}$, et une asthénozoospermie lorsque le pourcentage de spermatozoïdes mobiles était inférieur à $50 \%$ une heure après le recueil.

\section{RESULTATS et DISCUSSION}

\section{Caractéristiques générales et reproductives}

La tranche d'âge de 30 à 39 ans était la plus représentée (Tableau 1). C'est à cet âge effectivement que les hommes
Tableau 1 : Répartition des patients selon l'âge.

\begin{tabular}{lcc}
\hline Age (ans) & Nombre & $\%$ \\
$20-29$ & 268 & 11,9 \\
$30-39$ & 1275 & $\mathbf{5 6 , 7}$ \\
$40-49$ & 585 & 26 \\
$50-59$ & 104 & 4,6 \\
60 ans et plus & 18 & $\mathbf{0 , 8}$ \\
\hline Total & $\mathbf{2 2 5 0}$ & $\mathbf{1 0 0}$
\end{tabular}

commencent à prendre au sérieux le besoin d'enfant et aussi à se sentir concerné par le problème d'infertilité. Jusque là, ils pensent que c'est la femme qui est la seule responsable donc elle seule est concernée par la recherche des solutions et par le traitement. D'autres auteurs ont trouvé des résultats similaires $[3,4,6]$. Le désir d'enfant est exprimé même à un âge très avancé.

Trente quatre pour cent des hommes ont un régime polygamique allant de 2 à 4 épouses. Sur les 2250 sujets, 1286 $(57,2 \%)$ sont mariés à une seule épouse (Tableau 2). Le taux élevé de polygames indique que les hommes cherchent la solution à leur infertilité dans le mariage d'une nouvelle épouse. C'est parmi les hommes polygames que le taux d'azoospermie est élevé $[7,16]$. Ceci se comprend dans la mesure où l'homme pense plutôt à prendre une nouvelle épouse " qui serait capable " de lui donner un enfant que de commencer les explorations médicales chez lui [7]. Cette idée est d'ailleurs soutenue par la famille de l'homme qui lui suggère d'épouser une autre femme si celle avec qui il est ne lui donne pas d'enfant. Chez les polygames, la durée moyenne de vie commune avant de contracter un second mariage était très variable (4 à 15 ans), ainsi que le délai observé avant d'entamer les investigations spermiologiques dans notre population.

Tableau 2 : Répartition des patients selon le statut matrimonial

\begin{tabular}{lcc}
\hline Statut matrimonial & Nombre & $\%$ \\
Célibataire & 200 & 8,9 \\
Monogame & 1286 & 57,2 \\
Polygame & 764 & 33,9 \\
\hline Total & $\mathbf{2 2 5 0}$ & $\mathbf{1 0 0}$
\end{tabular}

Il existe une prédominance de la stérilité primaire $(70,4 \%)$. Ce taux est inférieur à celui retrouvé par Bakondé [3] qui est de $90 \%$. Cette prédominance pourrait s'expliquer par le fait que les couples en situation de stérilité primaire se remettent tôt en cause, plus que les couples présentant une stérilité secondaire. La première préoccupation après le mariage, c'est l'arrivée d'un enfant.

Plus le temps passe, plus les pressions familiales et sociales pèsent sur le couple en général et la femme en particulier. Celle-ci commence aussitôt les consultations sans son époux. Ce dernier a tendance à incriminer la femme. 


\section{Antécédents}

Dans notre étude, la bilharziose urinaire était l'antécédent infectieux le plus représenté (Tableau 3). Ceci prouve que la presque totalité des hommes ont fait une infection bilharzienne surtout à l'adolescence (10-19 ans). Au Mali, la bilharziose est un problème majeur de santé publique avec $25 \%$ de la population infectée [18]. L'origine bilharzienne de l'infertilité masculine n'est pas assez documentée. Dans la littérature, c'est surtout l'infertilité féminine qui est citée. Chez nos patients, $35,6 \%$ ont un antécédent de bilharziose et $28,5 \%$ avaient déjà contracté une gonococcie. Plusieurs auteurs ont rapporté le lien entre antécédents d'urétrite gonococcique et stérilité $[8,11,13]$.

Tableau 3 : Répartition des patients selon les antécédents infectieux.

\begin{tabular}{lcc}
\hline Antécédents infectieux & Nombre & $\%$ \\
Bilharziose & 803 & 35,6 \\
Gonococcie & 643 & 28,5 \\
Autres IST & 9 & 0,4 \\
Sans antécédent infectieux & 795 & 35,5 \\
\hline Total & $\mathbf{2 2 5 0}$ & $\mathbf{1 0 0}$
\end{tabular}

Plus de la moitié de nos patients (66\%) n'avaient aucun antécédent médical (Tableau 4). Par contre, les oreillons ont été l'antécédent médical le plus retrouvé. Il était généralement isolé $(14 \%)$ et quelques fois associé à un diabète ou à un traumatisme testiculaire. C'est une maladie virale à poussée épidémique sévissant surtout dans les collectivités et contractée dans l'enfance.

Tableau 4 : Répartition des patients selon les antécédents médicaux.

\begin{tabular}{lcc}
\hline Antécédents médicaux & Nombre & $\%$ \\
Traumatisme testiculaire & 52 & 2,3 \\
Orchite & 152 & 7,7 \\
Oreillons & 315 & 14 \\
Autres & 225 & 10 \\
Sans antécédents médicaux & 1506 & 66 \\
\hline Total & $\mathbf{2 2 5 0}$ & $\mathbf{1 0 0}$
\end{tabular}

\section{Spermoculture}

Les résultats de la spermoculture chez nos patients ont montré que $67,7 \%$ des cultures étaient stériles contre $32,3 \%$ de cultures positives. Les germes identifiés étaient : Staphylocoques aureus (16,3\%), Enterobacter agglomerus $(4,7 \%)$, E. coli $(7 \%)$, Klebsiella (4,7\%). Mogra et al. [14] ont trouvé des germes pathogènes chez $42,9 \%$ de leur population de 70 hommes. Des staphylocoques et des streptocoques étaient présents dans $65 \%$ des cas. Une infection non spécifique du tractus séminal pourrait être une importante cause d'infertilité masculine [8]. Les infections peuvent affecter la fertilité de plusieurs façons : altération du sperme en modifiant la mobilité des spermatozoïdes et la composition chimique du liquide séminal ou en provoquant un processus inflammatoire dans le tractus génital de l'homme. Ogunbanjo et al. [15] ont par ailleurs trouvé que $7 \%$ de leurs patients avaient une infection causée par divers germes.

\section{Paramètres spermatiques}

Dans notre étude $52 \%$ des patients avaient un volume de sperme normal (2-6 ml), 44\% avaient un volume inférieur à $2 \mathrm{ml}$ et $4 \%$ présentaient une hyperspermie. Bornman et al. [2] ont trouvé $45 \%$ d'hypospermie et $9 \%$ d'hyperspermie. Faute de n'avoir pu faire le dosage des marqueurs biochimiques, nous ne saurions malheureusement donner plus de précisions sur les causes de l'hypospermie (obstruction ou absence des canaux...).

La moitié de nos patients présentait une oligoasthénozoospermie modérée ou sévère $(49,5 \%)$. Parmi nos 2250 patients, $23,7 \%$ présentaient une azoospermie. Cette valeur est inférieure à celle de Yeboah et al. [19] qui ont trouvé $30,8 \%$ de cas d'azoospermie dans un échantillon de 595 hommes africains infertiles. Par contre, Bornman et al. [2] ont rapporté un chiffre beaucoup plus bas (9\%) dans une population de 1726 noirs africains. Nous avons un taux d'azoospermie élevé, mais nous n'avons pu établir la différence entre les deux types d'azoospermie, c'est-à-dire sécrétoire (non obstructive) et excrétoire (obstructive) en raison, entre autres, de la non disponibilité de plateau technique nécessaire pour faire la biochimie séminale.

Parmi les causes multiples de l'azoospermie, nous pouvons citer les séquelles d'infections uro-génitales telles que la gonococcie, la bilharziose urinaire et d'autres infections sexuellement transmissibles qui représentent $64,5 \%$ des antécédents infectieux chez nos patients. Dans la littérature, il existe très peu de documents sur l'implication directe de la bilharziose urinaire dans l'infertilité masculine $[11,17]$. Selon les résultats d'une étude préliminaire que nous avons effectué en zone hyper endémique et non endémique de bilharziose au Mali, nous avons constaté que le taux d'infertilité du couple était plus élevé en zone hyper endémique $[5,17,18]$. Comme d'autres auteurs, les résultats d'une de nos études précédentes ont montré que les lésions histologiques de la bilharziose étaient localisées sur les testicules dans $82,9 \%$ des cas, l'épididyme dans $14,3 \%$ et la prostate dans $2,9 \%$ des cas $[13,17]$.

\section{Bilan hormonal}

Les dosages hormonaux ont peu de valeur dans l'infertilité masculine, mais sont essentiels en cas d'azoospermie ou d'oligozoospermie sévère. Le dosage hormonal aide à déterminer si le sujet a une déficience gonadotrophique, une insuffisance testiculaire ou une défaillance de la spermatogenèse. La majorité des hommes infertiles a un taux normal de testostérone, de LH et de FSH. Dans les azoospermies obstructives, les taux de testostérone de LH et de FSH sont normaux $[1,20]$ et celui de la FSH est élevé dans les azoospermies non obstructives. 
Emokpae et al. [10], en déterminant le taux de trois hormones (testostérone, FSH et LH) chez 80 hommes infertiles présentant une azoospermie constitutive, ont trouvé une valeur anormale de ces hormones. Kuku et al. [12] ont trouvé que $59,9 \%$ des hommes étudiés avaient une valeur anormale de l'une ou plusieurs de ces hormones.

En déterminant le taux de FSH chez nos 534 sujets azoospermiques, nous avons observé que $63 \%$ avaient un taux élevé (>12 $\mathrm{mU} / \mathrm{ml}$ ) et $37 \%$ des valeurs comprises dans la normale $(1,7-12,0 \mathrm{mU} / \mathrm{ml})$. Le taux élevé de FSH nous orienterait vers une azoospermie non obstructive. Malheureusement, la biochimie séminale n'a pas été effectuée chez tous ces sujets dont le taux de FSH a été déterminé.

Chez nos 2250 patients, $48 \%$ avaient une valeur de LH élevée $(>7 \mathrm{mU} / \mathrm{ml})$ contre $52 \%$ qui avaient une valeur normale $(1,1-$ $7,0 \mathrm{mU} / \mathrm{ml}$ ). Aucun d'entre eux n'avait une valeur de $\mathrm{LH}$ inférieure à la normale. En ce qui concerne la prolactine, $33 \%$ de nos 2250 patients avaient un taux plus élevé que la normale ( $>450 \mathrm{mU} / \mathrm{L}$ ) contre $67 \%$ qui avaient un taux normal (< $450 \mathrm{mU} / \mathrm{L}$ ). Aucun des patients n'avait un taux bas de prolactine.

Dix neuf pour cent $(n=101)$ de nos sujets azoospermiques (Tableau 5) ont une valeur de testostérone inférieure à la normale $(3,0-10,6 \mathrm{ng} / \mathrm{ml})$. Ces 101 sujets présentaient tous une hypospermie, ce qui nous a fait suggérer que ceux-ci constituaient des cas d'hypogonadisme. L'hypogonadisme est caractérisé par le déficit de testostérone entraînant une azoospermie avec hypospermie.

Tableau 5 : Répartition des patients azoospermiques selon le taux de testostérone.

\begin{tabular}{lcc}
\hline Testostérone (ng/ml) & Nombre & $\%$ \\
$3-10,6$ & 412 & 77 \\
$>10,6$ & 21 & 4 \\
$<3$ & 101 & 19 \\
\hline Total & $\mathbf{5 3 4}$ & $\mathbf{1 0 0}$
\end{tabular}

\section{CONCLUSION}

L'infertilité masculine est un problème de santé publique au Mali. La prise en charge est retardée par le fait que les unions d'une part, et la prise de décision de commencer les investigations chez l'homme, sont tardives. Les causes en sont multiples. Le spermogramme reste, à l'heure actuelle, la pierre angulaire de l'évaluation de l'infertilité masculine. La tranche d'âge la plus touchée se situe entre 30 et 39 ans. La stérilité primaire est fortement représentée par $70 \%$ des sujets étudiés. La bilharziose occupe une place prédominante dans les antécédents infectieux. Les résultats du spermogramme montrent que les cas d'oligo-asthénospermie et d'azoospermie sont assez nombreux dans notre population étudiée.

L'examen spermiologique de base (spermogramme) doit être envisagé en premier lieu dans un pronostic d'infertilité masculine. Les examens biologiques complémentaires (biochimie, dépistage des anticorps anti-spermatozoïdes, dosage hormonaux) sont souvent indispensables pour un meilleur diagnostic. Malheureusement ces examens ne sont pas encore réalisés de façons régulières dans le service. Il est donc impératif de mettre ces examens dans la routine. Pour cela il faudrait établir une plus grande collaboration entre médecins généraliste, gynécologue, endocrinologue, urologue, biologiste, pour un meilleur confort des patients et/ou couples affectés par le problème d'infertilité.

Le dosage des hormones et des marqueurs biochimiques doit être systématique devant une azoospermie pour faire le diagnostic différentiel entre une azoospermie sécrétoire et une azoospermie obstructive. L'organisation des séances de formation continue des médecins et des biologistes en biologie de la reproduction et en andrologie s'avère nécessaire pour une meilleure prise en charge de l'infertilité masculine.

\section{ABSTRACT}

Sperm profile of infertile men in Mali

\author{
Mahamadou TRAORE, Amadou TOURE, Sidi SISSOKO, \\ N'Dia Fatoumata SAMAKE
}

Infertility remains a major sociocultural problem in view of the fact that in Africa, in general, and in Mali, in particular, the main purpose of marriage remains procreation. The birth of a child is a source of joy and contributes to maintenance of a certain harmony in the home.

In Africa, infertility is a dramatic problem that is difficult to bear for the couple. It is the leading cause of marital discord or divorce. It is considered to be due to fate, or a curse inflicted on the couple. For the woman, it represents a divine punishment, as it is usually the woman who is blamed for the problem male responsibility is only discovered during assessment of the couple's infertility.

Sperm count and sperm cytology are the basic examinations performed on semen. These examinations constitute key elements to evaluate the quality of semen. In Mali, the Cytogenetics and Reproduction Biology laboratory performs the greatest number of semen examinations in the country.

The purpose of this study was to present the sperm profile of infertile men in Mali based on a 5-year retrospective study. Male infertility is a public health problem in Mali. The agegroup most frequently affected is between 30 and 39 years. Primary sterility was the predominant form, observed in $70 \%$ of the subjects studied. Schistosomiasis plays a predominant role in the infectious history. The sperm count results show that cases of oligoasthenozoospermia and azoospermia represent $49.5 \%$ and $23.7 \%$ of the study population, respectively.

Key-Words : sperm count, male infertility, infectious history, azoospermia 


\section{REFERENCES}

1. BHASIN S. : Approach to the infertile man. J. Clin. Endocrinol. Metab., 2007, 92 : 1995-2004.

2. BORNMAN M.S., SCHULENBURG G.W., BOOKER D., CHAUKE T.R., REIF S. : Observations in infertile African males at an andrology clinic in South Africa. Arch. Androl., 1994, 33 : 101-104.

3. BAKONDE B. : Contribution à l'étude de la stérilité masculine. Thèse Méd., 1983, n¹0, Dakar.

4. KAHAM PENLAP C. :Analyses cytospermiologiques au service de cytogénétique et de biologie de la reproduction de L'INRSP. A propos de 860 cas. Thèse Méd., 2005, $n^{\circ} 25$, FMPOS, Bamako.

5. CISSÉ H.L. : Contribution à l'étude de la relation entre bilharziose urinaire et stérilité du couple. Thèse Méd., 2007, FMPOS, Bamako.

6. CISSÉ C.T., DAO B., AFOUTOU J.M., MENSAH A. : Stérilité conjugale au CHU de Dakar. Profil épidémiologique et limites du bilan. Vie et Santé, 1992, 12 : 15-18.

7. COULIBALY A.O. : Caractéristiques cytospermiologiques de la stérilité masculine : à propos de 598 examens. Thèse Méd., 2001, $n^{\circ} 107$, FMPOS, Bamako.

8. DIARRA S., TOURE A., TRAORÉ M. : Aspect bactériologique et parasitologique de 200 prélèvements de sperme. Mali Méd., 1997, $12: 24-26$.

9. ELZANATY S., MALM J., GIWERCMANA. : Visco-elasticity of seminal fluid in relation to the epididymal and accessory sex gland function and its impact on sperm motility. Int. J. Androl., 2004, $27: 94-100$.

10. EMOKPAE M.A., UADIAP.O., MOHAMMEDA.Z., OMALE-HODOA.: Hormonal abnormalities in azoospermic men in Kano, Northern Nigeria. Indian J. Med. Res., 2006, 124 : 299-304.

11. FELDMEIR H., LEUTSCHER P., PONENESEE G., HARMS G. : Male genital schistosomiasis and haemospermia. Trop. Med. Int. Health, 1999, 4 : 791-793.

12. KUKU S.F., AKINYANJU P.A., OJEIFO J.O. : Serum levels of gonadotropins, prolactin, and testosterone in oligo/azoospermic Nigerian males. Int. J. Fertil., 1988, 33 : 40-44.

13. MALONGA G., SILOU J., LOCKO-MAFOUTA C. : Facteurs étiologiques de la stérilité conjugale en Afrique Noire. Méd. Af. Noire, 1986, $33: 721-730$.

14. MOGRAN., DHRUVAA., KOTHARI LK. : Non-specific seminal tract infection and male infertility : a bacteriological study. J. Postgrad. Med., 1981, $27:$ 99-104.

15. OGUNBANJO B.O., OSOBAA.O., OCHEI J. : Infective factor of male infertility among Nigerians. Afr. J. Med. Sci., 1989, $18: 35-38$.

16. TOURÉ A., TRAORÉ M. : Aspects socio-démographiques et biologiques de la stérilité masculine à Bamako (à propos de 200 cas). Mali Méd., 1996, $11:$ 31-33.

17. TOURÉ A., TRAORÉ M., SACKO M., KAMATÉ D., COULIBALY C., BAYO S. : Bilharziose à schistosoma haematobium du tractus génital de l'homme : à propos de 35 cas. Mali Méd., 2000, $15: 45-46$.

18. TRAORE M., TRAORE H.A, KARDOFF R. et al. : The public health significance of urinary schistosomiasis as a cause of morbidity in two districts in Mali. Am. J. Trop. Med. Hyg., 1998, 59 ; 407-413.

19. YEBOAH E.D., WADHWANI J.M., WILSON J.B. : Etiological factors of male infertility in Africa? Int. J. Fertil., 1992, $37: 300-307$.

20. WEISS D.B., GOTTSCHHALK-SABAG S., ZUKERMAN Z., BARON E., KAHANA Z. : Follicle-stimulating hormone in azoospermic in prediction of spermatogenic patterns. Harefuah, 1998, 135 : 169-175.

21. VEXIAU P., BOUDOU P., BRERAULT J.L. et al. : Hyperandrogénie clinique et stérilité. Immunoanal. Biol. Spéc., 1990, 22: 35- 40.

22. WORLD HEALTH ORGANIZATION: WHO Laboratory Manual for the Examination of Human Semen and Semen-Cervical Mucus Interactions, 4th edition, 1999. 